\title{
Mode Switching in Red Semiregular Variable Stars?
}

\author{
J. Gál and K. Szatmáry \\ Astron. Obs. and Dept. of Exp. Physics, JATE University, Dóm tér 9, \\ H-6720, Szeged, Hungary, Internet: J.Gal@physx.u-szeged.hu
}

\begin{abstract}
Light curve analysis of the carbon variable RY Dra and the oxygen-rich variables TX Dra and AF Cyg is summarised. In each case, two shorter periods were found (which may be connected with the radial pulsation of the stars) and also a long term variation in the mean brightness. In the case of RY Dra, the amplitudes of the shorter periods both decreased while the amplitude of the long secondary variation was found to be increasing. The light curves and frequency spectra of TX Dra and AF Cyg can be interpreted as a result of mode switching. These stars pulsate with two different periods and there are time intervals when the longer period disappears and the shorter one remains dominant, and it lasts for many cycles. We concluded that the double and multi-periodic red semiregular variables may be very important from both theoretical and observational point of view, because, based on the ratio of the periods, we can identify the pulsational modes in these variables.
\end{abstract}

Semiregular variables are red giant or supergiant stars with spectral types $\mathrm{M}, \mathrm{C}$ or S. They are located on the Asymptotic Giant Branch of the H-R Diagram.

Here we present a short summary of the light curve analysis of three interesting red variables. Due to the lack of photometric data, we have used visual observations taken from different data bases. For the light curve analysis we constructed mean light curves by averaging the data in 10-day bins. The errors of these mean points were generally $0.2 \mathrm{mag}$. It is important to note that the observations from the different sources did not show systematic differences.

The periods were determined using the well-known discrete Fourier transformation. Since the periods and the amplitudes are subject to variations in all three program stars the windowed Fourier transformation was used to study the different parts of the light curves.

In the case of RY Dra the mean light curve contains 1079 points between JD 2433880 and 2449560 . Unfortunately there is a long gap (JD 2437600-41930) with only a few observations. The data were divided into three segments (based on the light curve shape) and these segments were analysed separately. In the first part (JD 2433880-2437600) two short periods are detectable: 259.3 and 155.4 days. The shape of the light curve is rather different about four thousand days later which means that the amplitudes of the short periods decrease and a new slow oscillation develops; its characteristic time scale is around 900 days. After JD 2446000 the light curve is dominated by a slow variation. 


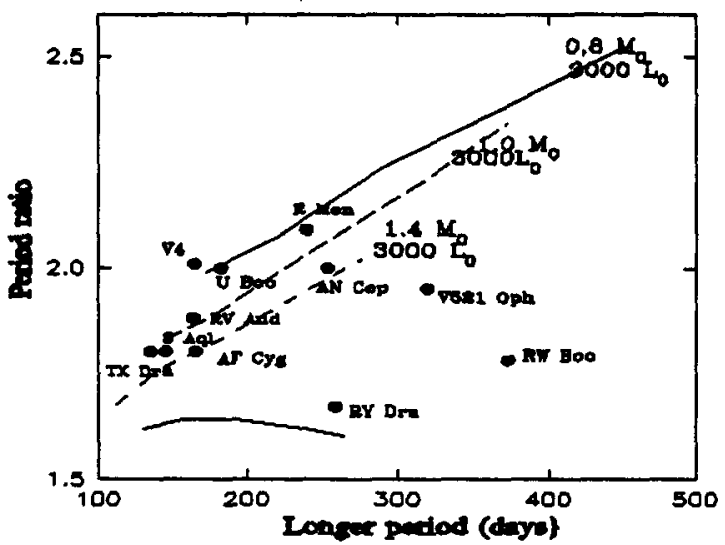

Figure 1. Longer period vs. period ratio diagram for some red semiregular stars. The lines represents the model calculations of Ostlie $\&$ Cox

The other two stars (TX Dra and AF Cyg) show a rather complex light variation. The shape of the light curves are strongly variable. In both cases there are time intervals where the brightness is approximately constant. It is important to note that TX Dra has a stable long secondary variation (700 days) which is probably not due to pulsation.

Due to the limited space the detailed description of the light curves is not presented here. The light curves are characterised by two short periods; however the amplitudes of these periods are strongly variable. In fact there are time intervals where both short periods are present and there are time intervals where the longer period disappears and the shorter one remains dominant, and it lasts during many cycles. These light variations may be connected with mode switching.

Mode switching was found in three red semiregular variables by Cadmus et al. (1991), although the general characteristic of light variation of Cadmus's stars are different from our program stars.

Since all three variables have two short periods which may connected with the radial pulsations of the stars, based on period ratios and model calculations of Ostlie \& Cox (1986) we can identify these periods as pulsational modes. In Fig. 1 we plotted the period ratio as a function of the longer period. The lines represent the models of Ostlie \& Cox. The figure suggests that the fundamental and the first overtone modes may be excited in these variables.

Acknowledgments. This work was supported by the Hungarian OTKA Grants No. T7522 and F7318.

\section{References}

Cadmus, R.R., Willson, L.A., Sneden, C., \& Mattei, J.A. 1991, AJ, 101, 1043 Ostlie, D.A., \& Cox, A.N. 1986, ApJ, 311, 864 